\title{
Highly-accurate long-read sequencing improves variant detection and assembly of a human genome
}

\section{Authors}

Aaron M. Wenger ${ }^{1} \dagger$, Paul Peluso ${ }^{1} \dagger$, William J. Rowell ${ }^{1}$, Pi-Chuan Chang ${ }^{2}$, Richard J. Hall ${ }^{1}$, Gregory T. Concepcion ${ }^{1}$, Jana Ebler ${ }^{3,4,5}$, Arkarachai Fungtammasan ${ }^{6}$, Alexey Kolesnikov ${ }^{2}$, Nathan D. Olson ${ }^{7}$, Armin Töpfer ${ }^{1}$, Michael Alonge ${ }^{8}$, Medhat Mahmoud ${ }^{9}$, Yufeng Qian ${ }^{1}$, Chen-Shan Chin ${ }^{6}$, Adam M. Phillippy ${ }^{10}$, Michael C. Schatz ${ }^{8}$, Gene Myers $^{11}$, Mark A. DePristo ${ }^{2}$, Jue Ruan ${ }^{12}$, Tobias Marschall ${ }^{3,4}$, Fritz J. Sedlazeck ${ }^{9}$, Justin M. Zook ${ }^{7}$, Heng Li ${ }^{13}$, Sergey Koren ${ }^{10}$, Andrew Carroll ${ }^{2}$, David R. Rank ${ }^{1 *}$, Michael W. Hunkapiller $^{1 *}$

† These authors contributed equally to this work.

* Address correspondence to M.W.H. (mhunkapiller@pacb.com) or

D.R.R. (drank@pacb.com).

1. Pacific Biosciences, Menlo Park, CA, USA

2. Google Inc., Mountain View, CA, USA

3. Center for Bioinformatics, Saarland University, Saarbrücken, Germany

4. Max Planck Institute for Informatics, Saarland Informatics Campus E1.4, Saarbrücken, Germany

5. Graduate School of Computer Science, Saarland University, Saarland Informatics Campus E1.3, Saarbrücken, Germany

6. DNAnexus, Mountain View, CA, USA

7. National Institute of Standards and Technology, Gaithersburg, MD, USA

8. Department of Computer Science, Johns Hopkins University, Baltimore, MD, USA

9. Human Genome Sequencing Center, Baylor College of Medicine, Houston, TX, USA

10. Genome Informatics Section, Computational and Statistical Genomics Branch, National Human Genome Research Institute, Bethesda, MD, USA

11. Max Planck Institute of Molecular Cell Biology and Genetics, Dresden, Germany

12. Agricultural Genomics Institute, Chinese Academy of Agriculture Sciences, Shenzen, China

13. Dana-Farber Cancer Institute, Boston, MA, USA 


\section{Abstract}

The major DNA sequencing technologies in use today produce either highly-accurate short reads or noisy long reads. We developed a protocol based on single-molecule, circular consensus sequencing (CCS) to generate highly-accurate $(99.8 \%)$ long reads averaging $13.5 \mathrm{~kb}$ and applied it to sequence the well-characterized human HG002/NA24385. We optimized existing tools to comprehensively detect variants, achieving precision and recall above $99.91 \%$ for SNVs, $95.98 \%$ for indels, and $95.99 \%$ for structural variants. We estimate that 2,434 discordances are correctable mistakes in the high-quality Genome in a Bottle benchmark. Nearly all (99.64\%) variants are phased into haplotypes, which further improves variant detection. De novo assembly produces a highly contiguous and accurate genome with contig N50 above $15 \mathrm{Mb}$ and concordance of $99.998 \%$. CCS reads match short reads for small variant detection, while enabling structural variant detection and de novo assembly at similar contiguity and markedly higher concordance than noisy long reads.

\section{Introduction}

DNA sequencing technologies have improved at rates eclipsing Moore's law ${ }^{1}$ revolutionizing biological sciences. Beginning in the 1970s, Sanger sequencing ${ }^{2}$, and subsequent automation ${ }^{3}$ facilitated large scale DNA sequencing projects and paved the way for modern genomic research ${ }^{4-7}$. The first reference genomes were followed by the advent of several high-throughput sequencing technologies (next-generation sequencing or NGS) including $454^{\mathrm{TM}}$, Solexa/lllumina ${ }^{\circledR}, \mathrm{ABI}^{\circledR}$ Solid ${ }^{\mathrm{TM}}$, Complete Genomics $^{\mathrm{TM}}$, and lon Torrent ${ }^{\mathrm{TM}}$. These technologies employed a range of chemistries and detection strategies ${ }^{8-13}$. All produce relatively accurate reads but are limited in read length, typically to less than 300 basepairs (bp). These accurate short reads are wellsuited for calling single-nucleotide variants (SNVs) and small insertions and deletions (indels), but are lacking for long-range applications such as de novo assembly, haplotype phasing, and structural variant detection.

For these applications, vastly superior results ${ }^{14-18}$ are obtained with technologies like $\mathrm{PacBio}^{\circledR}$ SMRT Sequencing ${ }^{19}$ and Oxford Nanopore sequencing ${ }^{20}$ that produce long reads $(>10 \mathrm{~kb})$. These technologies rely on single-molecule detection and are characterized by reduced read accuracy $(75-90 \%)^{19,20}$. High consensus accuracy has been demonstrated through read-to-read error correction, but the process is computationally intensive, and errors remain from mis-mapping reads and mixing haplotypes during correction ${ }^{15,21}$. As a result of the error rate, long-read technologies are rarely used to detect SNVs and indels.

Today, human genomes are sequenced at population scales, but it remains necessary to combine sequencing technologies to cover all types of genetic variation, which increases cost and adds complexity to projects. A sequencing technology with long 
read length and high accuracy would enable a single experiment for comprehensive variant discovery.

Recent gains in read length for SMRT Sequencing and optimized DNA template preparation suggested an opportunity to unify high accuracy with long read lengths using circular consensus sequencing (CCS) ${ }^{22,23}$. CCS derives a consensus sequence from multiple passes of a single template molecule, producing accurate reads from noisy individual subreads.

Here, we highlight the performance of these highly-accurate, long $\mathrm{CCS}^{\mathrm{TM}}$ reads by sequencing and analyzing the well-characterized human male HG002/NA2438524,25. The HG002 sample is one of the benchmark samples available from the Genome in a Bottle (GIAB) Consortium. GIAB provides physical reference materials along with detailed characterization of the sample genome, defining "high-confidence regions" at which the sequence of the sample is known and "high-confidence variants" within those regions at which the sample differs from the human reference genome. Thus, it is an ideal sample for study of sequencing accuracy and variant detection. We apply and extend standard analysis tools to identify variation in $\mathrm{HG} 002$, demonstrating performance that rivals or surpasses existing technologies for small and large variation detection as well as genome assembly and haplotype phasing.

\section{Results}

\section{CCS Library Preparation and Sequencing}

A SMRTbell library tightly distributed at $15 \mathrm{~kb}$ was chosen for circular consensus sequencing (Figure 1a, Supplementary Figure 1) based on estimates of $150 \mathrm{~kb}$ polymerase read length and a requirement of 10 passes to achieve Q30 read accuracy (Figure 1b). CCS reads with a predicted accuracy of at least Q20 (99\%) were retained (Supplementary Figure 2a). The total CCS read yield was $89 \mathrm{~Gb}(2.3 \pm 0.4 \mathrm{~Gb}$ over 39 SMRT Cells), with read length of $13.5 \pm 1.2 \mathrm{~kb}$ (Figure 1c). The predicted accuracy of the CCS reads has a median of Q30 (99.9\%) and a mean of Q27 (99.8\%) (Figure 1c). Predicted accuracy matches well with concordance to the GIAB HG002 benchmark (average $\left[Q_{\text {predicted }}-Q_{\text {concordance }}\right]=-1.2$ ), which indicates that the predicted accuracy is well calibrated (Supplementary Figure $\mathbf{2 b - c )}$ ). Average mapped coverage of the genome is 28-fold, with minimal difference across [GC] content (Supplementary Figure 2d-e).

\section{Quality Evaluation of CCS Reads}

To characterize the few residual errors in CCS reads, discordances between the reads and the GIAB HG002 benchmark were tallied. The average read concordance is $99.8 \%$, comparable to the $99.9 \%$ concordance of short reads from the lllumina NovaSeq (Supplementary Table 1). The large majority of CCS read discordances are indels in homopolymer contexts: $3.4 \%$ are mismatches, $4.6 \%$ are indels in non-homopolymer contexts, and $92.0 \%$ are indels in homopolymers. This equates to a mismatch every 
13,048 bp in CCS reads, a non-homopolymer indel every 9,669 bp, and a homopolymer indel every $477 \mathrm{bp}$ (Supplementary Table 1). The mismatch rate is $17 \times$ lower than reads from the Illumina NovaSeq, while the indel rate is $181 \times$ higher (Supplementary Table 1).

To confirm independently the high quality of CCS reads, error rates were measured through read-to-read alignments ${ }^{26}$. Consistent with the reference-based methods, the average read accuracy is estimated at $99.8 \%$. A putative large artifact is detected in $0.6 \%$ of reads: $0.5 \%$ are molecular chimeras, likely due to ligation of DNA fragments during library construction, $0.1 \%$ contain a "low quality" run of bases, anecdotally in microsatellites, and $0.03 \%$ have a missing SMRTbell adapter on one end. Overall, the read-to-read comparison supports the predicted quality of the CCS reads.

\section{Increased Mappability of CCS Reads}

To evaluate increases in mappability with long reads, the $13.5 \mathrm{~kb}$ CCS reads and a coverage-matched number of $2 \times 250$ bp NGS short reads were mapped to GRCh37. A genomic position was considered to be mappable if it is covered by least ten reads. At the highest reported mapping quality (60), 97.5\% of the non-gap GRCh37 is mappable with $13.5 \mathrm{~kb}$ CCS reads, while $94.8 \%$ is mappable with NGS short reads (Figure 2a).

The additional regions that are now accessible with longer CCS reads include numerous medically-relevant genes which have been previously reported as recalcitrant to NGS sequencing ${ }^{27}$. Of the 193 reported medically-relevant genes with at least one NGS problem exon, 152 are fully mappable with the CCS reads, including CYP2D6, GBA, PMS2, and STRC (Figure 2b-c).

The $13.5 \mathrm{~kb}$ CCS reads also resolve complex regions, like the HLA class 1 and 2 genes, which are fully phased and typed to four-field resolution ${ }^{28}$ (Supplementary Figure 3).

\section{Small Variant Detection with CCS Reads}

GATK $^{29}$ was used to call SNVs and small indels with CCS reads. Evaluated against the GIAB benchmark ${ }^{25}$, precision for SNVs is $99.468 \%$ and recall is $99.559 \%$. For indels, precision is $78.977 \%$ and recall is $81.248 \%$. While GATK performance with CCS reads is comparable to NGS for SNVs, it is lower for indels (Table 1). Unlike NGS read errors, which are mostly mismatches, CCS read errors are mostly indels

(Supplementary Table 1), contributing to the low indel precision and recall of GATK for CCS reads.

Variant callers based on deep learning have an inherent ability to adapt to the error profiles of new data types. To evaluate variant calling with a deep learning framework, Google DeepVariant ${ }^{30}$ was used to call SNVs and indels from CCS reads. Using a model trained on Illumina reads, precision is $99.533 \%$ and recall is $99.793 \%$ for SNVs, and precision is $23.991 \%$ and recall is $81.692 \%$ for indels (Supplementary Table 2). 
Training a model on CCS reads provides a large boost in precision and recall for both SNVs and indels. For SNVs, DeepVariant achieves precision of $99.914 \%$ and recall of 99.959\%. For indels, DeepVariant achieves $96.901 \%$ precision and $95.980 \%$ recall (Figure 3a, Table 1). Most discordant indels (90.33\%) occur in homopolymer runs, matching the most common discordance in CCS reads (Supplementary Figure 4). The callset includes 1,969 SNVs and 62 indels in exons of medically-relevant genes previously reported as recalcitrant to NGS sequencing.

\section{Phasing Small Variants with CCS Reads}

To determine whether CCS reads could provide both highly-accurate variant calls and long-range information needed to generate haplotypes, we used WhatsHap ${ }^{31}$ to phase the DeepVariant variant calls. Nearly all (99.64\%) autosomal heterozygous variants were phased into 19,215 blocks with an N50 of 206 kb (Supplementary Table 3). The phase block length distribution closely matches the theoretical limit estimated by creating breaks between variants that are separated by more than the average CCS read length of $13.5 \mathrm{~kb}$. This suggests that the phase block length is limited by read length and the amount of variation in $\mathrm{HG} 002$, not by coverage or the quality of the variant calls (Figure $\mathbf{3 b}$, Supplementary Figure 5). Evaluated against the GIAB benchmark phase set, the switch error rate is $0.37 \%$ and the Hamming error rate is 1.91\% (Supplementary Table 3).

\section{Improving Small Variant Detection with Haplotype Phasing}

GATK and DeepVariant do not directly incorporate long-range haplotype phase information when calling variants. To evaluate whether phase information improves results, CCS reads were haplotype-tagged based on trio-phased variants from GIAB (which tags $84.55 \%$ of reads) and a DeepVariant model was then trained on reads passed in haplotype-sorted order. The haplotype-sorted model performs similarly to the original DeepVariant CCS model for SNVs but provides a large improvement for indels achieving precision of $97.835 \%$ and recall of $97.141 \%$ (Table 1 ).

\section{Structural Variant Detection with CCS Reads}

Insertion and deletion structural variants $\geq 50$ bp were called using two read mappingbased tools, pbsv (https://github.com/PacificBiosciences/pbsv) and Sniffles ${ }^{32}$. The callsets show similar precision (>94\%) and recall (>91\%) against the GIAB benchmark (Supplementary Table 4). Precision is consistent across variant length, but recall is lower for variants $\geq 3 \mathrm{~kb}$ (Supplementary Figure 6a-b). To increase recall for larger variants, haplotype-resolved de novo assemblies were analyzed with paftools ${ }^{33}$ (see "De Novo Assembly of CCS Reads"), with precision $>93 \%$ and recall $>89 \%$ (Supplementary Figure 6c-d, Supplementary Table 4).

An integrated callset includes 8,432 deletions and 12,091 insertions. Precision is $96.13 \%$ and recall is $95.99 \%$ (Figure 3c, Supplementary Table 4), with similar performance for insertions as deletions and for variants $<1 \mathrm{~kb}$ as $\geq 1 \mathrm{~kb}$ (Figure $3 \mathrm{~d}$ ), 
indicating the complementarity of mapping- and assembly-based structural variant calling. The callset has 163 deletions and 143 insertions that intersect exons.

For comparison, structural variants were called in Illumina $2 \times 250$ bp short reads (with Manta $^{34}$ and Delly ${ }^{35}$ ) and 10X Genomics linked reads (with LongRanger ${ }^{36}$ ) available from $\mathrm{GIAB}^{24}$. The Manta callset has precision of $85.34 \%$ and recall of $55.88 \%$, with much worse recall for insertions (39.65\%) than deletions $(76.90 \%)$. The LongRanger callset has precision of $83.79 \%$ and recall of $39.83 \%$, again with worse recall for insertions (16.41\%) than deletions (70.18\%). A callset from paftools run on a linkedread SuperNova assembly has precision of $64.52 \%$ and recall of $52.74 \%$

(Supplementary Table 4, Supplementary Figure 6e-h). All considered short- and linked-read callsets have worse performance than all CCS callsets in both precision and recall.

\section{De Novo Assembly of CCS Reads}

Three different algorithms - FALCON ${ }^{37}$, $\mathrm{Canu}^{38}$, and wtdbg2 (https://github.com/ruanjue/wtdbg2) - were used to assemble the full CCS read set, which is a mix of paternal and maternal reads. By skipping the initial read-to-read error correction step, the algorithms completed 10-100x faster than is typical for long-read assemblies $^{21}$ (Supplementary Table 5). All assemblies have high contiguity with a contig N50 from 15.43 to $28.95 \mathrm{Mb}$. The total assembly size is near the expected human genome size for FALCON and wtdbg2. The Canu assembly has a total genome size of $3.42 \mathrm{~Gb}$, larger than the expected haploid human genome, because it resolves some heterozygous alleles into separate contigs (Table 2, Supplementary Figure 7).

Short reads from the parents of HG002 were used to identify k-mers unique to one parent and then partition ("trio bin") the CCS reads by haplotype ${ }^{39}$. Three different k-mer sizes were evaluated: 21 bp (previously reported for trio binning) and longer kmers of $51 \mathrm{bp}$ and $91 \mathrm{bp}$ enabled by the accuracy of CCS reads. The 21-mer binning assigns $35.3 \%$ of reads to the mother and $33.6 \%$ to the father (68.9\% binned). The $51-$ mer binning is more complete at $78.5 \%$ binned; using longer 91 -mers provides only a small additional gain to $79.2 \%$ binned. The $51-$ mer binning was selected for assembly (Supplementary Table 6).

FALCON, Canu, and wtdbg2 were run separately on the paternal and maternal reads, with the unassigned reads included in both sets. All algorithms produce highly contiguous and nearly complete assemblies for the parental genomes, with N50 from 12.10 to $19.99 \mathrm{Mb}$ and genome size from 2.67 to $3.04 \mathrm{~Gb}$ (Table 2). From $95.3 \%$ to $98.2 \%$ of human genes are identified as single-copy in each parental assembly (Table 2). Assembly-based structural variant calls have high precision and recall, suggesting few large-scale mis-assemblies (Supplementary Table 4). Furthermore, analysis of the phase-consistency ${ }^{40}$ of maternal and paternal haplotigs shows the assemblies are phased properly (Supplementary Figure 8). 
All mixed and parental assemblies are high quality with concordance to the HG002 benchmark ranging from Q44-Q48 for polished ${ }^{41}$ and Q26-Q45 for unpolished assemblies (Table 2, Supplementary Table 7). This greatly exceeds that of previously published and accessioned assemblies at Q40 (6× worse) for PacBio noisy long reads and Q29 (77× worse) for Oxford Nanopore reads with Illumina polishing (Figure 4a, Supplementary Table 7).

Large segmental duplications often result in contig breaks in de novo assemblies, and assemblies of noisy long reads typically span less than 50 of $175 \mathrm{Mb}$ of segmental duplications in the human genome ${ }^{15,18,42}$. The most contiguous assemblies of CCS reads span over $60 \mathrm{Mb}$ of segmental duplications, a $20 \%$ improvement

(Supplementary Table 8). A model of assembly contiguity based on large repeat resolution suggests that the current assemblies of CCS reads resolve $15 \mathrm{~kb}$ repeats of 99 to $99.5 \%$ identity (Figure $\mathbf{4 b}$ ).

\section{Coverage Requirements for Variant Calling and De Novo Assembly}

To evaluate the depth of CCS read coverage required for variant calling and assembly, we randomly subsampled from the full dataset. For SNVs, precision and recall with DeepVariant remain above $99.5 \%$ for coverage down to 15 -fold; performance decays steeply below 10-fold (Supplementary Figure 9a). For indels, DeepVariant remains comparable to typical NGS performance (>90\%) down to 17-fold coverage

(Supplementary Figure 9b). For structural variants, precision with pbsv is above 95\% for all evaluated coverage levels. Recall is above $90 \%$ down to 15 -fold coverage, and decays steeply below 10-fold (Supplementary Figure 9c). For phasing with WhatsHap, the phase block N50 remains above $150 \mathrm{~kb}$ down to 10-fold coverage (Supplementary Figure 9d). Mixed-haplotype wtdbg2 assemblies have consistent size above $2.7 \mathrm{~Gb}$, contig N50 around $15 \mathrm{Mb}$, and concordance above Q42 until coverage falls below 15-fold (Supplementary Figure 9e-g).

\section{Revising and Expanding Genome in a Bottle Benchmarks}

High-quality callsets from CCS reads provide an opportunity to identify mistakes in the GIAB benchmarks, particularly for structural variants where the benchmark is still in draft form. Sixty small variant and 40 structural variant discrepancies between the GIAB benchmark (small variant v3.3.2, structural variant v0.6) and the CCS callsets

(DeepVariant haplotype-sorted, structural variant integrated) were selected for manual curation. Selected variants were spread across variant types, discrepancy types, and both inside and outside homopolymers and tandem repeats.

For small variants, 29 of 31 discrepancies in homopolymers were classified as correct in the benchmark. Outside of homopolymers, 19 of 29 were classified as errors in the benchmark. Most of these benchmark errors (13 of 19) are true variants in L1 elements called homozygous reference in GIAB (Supplementary Figure 10a-b, Supplementary Table 9). The identified benchmark errors overlap with putative errors in a DeepVariant Illumina whole genome case study (https://github.com/google/deepvariant). Of 745 
putative false positive SNVs in the case study, 344 agree with the CCS callset, with 282 $(82.0 \%)$ falling within large interspersed repeats. Fewer of the false negative SNVs $(8 \%)$, false negative indels (25\%), and false positive indels $(19 \%)$ from the case study agree with the CCS callset. Extrapolating from manual curation, we estimate that 2,434 $(1,313-2,611 ; 95 \%$ confidence interval) errors in the current GIAB benchmark could be corrected using the CCS reads.

For structural variants, curator classification was unclear for 11 of 40 discrepancies, typically because of tandem repeat structure that permits multiple representations of a variant. For the remainder, 15 of 16 false negative discrepancies were classified as correct in the benchmark. However, for false positive discrepancies, 11 of 13 were classified as errors in the benchmark (Supplementary Figure 10c-d, Supplementary Table 10). This suggests that the GIAB structural variant benchmark set is precise but incomplete.

The high-quality CCS callsets also provide an opportunity to expand the benchmarks into repetitive and highly polymorphic regions that have been difficult to characterize with confidence using short reads. Adding the CCS DeepVariant callset to the existing GIAB small variant integration pipeline would expand the benchmark regions by up to $1.3 \%$ and 418,875 variants (210,184 SNVs and 208,691 indels). For structural variants, only 9,232 of 18,832 autosomal variant calls overlap benchmark regions, which means that the number of variants in the benchmark would more than double if all CCS variants calls were incorporated.

\section{Discussion}

We present a protocol for producing highly-accurate long reads using circular consensus sequencing (CCS) on the PacBio Sequel System. We apply the protocol to sequence the human HG002 to 28-fold coverage with average read length of $13.5 \mathrm{~kb}$ and an average read accuracy of $99.8 \%$. We analyze the CCS reads to call SNVs, indels, and structural variants; to phase variants into haplotype blocks; and to de novo assemble the HG002 genome.

The CCS performance for SNV and indel calling rivals that of the commonly-used pairing of BWA and GATK on 30-fold short-read coverage. Interestingly, though the overall accuracy of CCS reads is similar to short reads, direct application of the GATK pipeline to CCS reads produces inferior results, especially for indels. The major residual error in CCS reads - indels in homopolymers - is not as frequent in short reads. We suspect that the current GATK, which was designed for short reads, does not properly model the CCS error profile, and thus performance lags for indels. This is supported by results with DeepVariant. When a DeepVariant model trained on Illumina reads is run on CCS reads, the performance is poor for indels. When DeepVariant is trained on CCS reads, performance improves dramatically. As more CCS datasets are made available, both model-based callers like GATK and learning-based callers like 
DeepVariant will have the opportunity to improve on the performance reported here, including by incorporating haplotype phase information and evaluating and training against updated GIAB benchmarks that correct errors with CCS reads. Further, advances in sequencing chemistry or consensus base calling (such as the application of deep learning) that reduce the residual indel errors in CCS reads also could improve variant calling performance.

Structural variant calling and de novo genome assembly with CCS reads match or exceed that reported for noisy long reads. The CCS reads have an advantage of high accuracy, which eliminates the need for read correction, allows more stringent criteria to be used in variant calling or read overlapping, and ultimately produces more accurate assemblies and variant calls. Noisy long reads have an advantage of longer maximum read length, but increased accuracy of CCS reads compensates for the length required for highly contiguous assembly. Modeling (Figure 4b) suggests modest advances in accuracy (to $99.9 \%$ ) at $15 \mathrm{~kb}$ read length would double the current contiguity, which already matches the best published de novo assemblies ${ }^{43}$.

The CCS read approach alleviates some other challenges of long-read sequencing. First, aiming for fragments in the $10-20 \mathrm{~kb}$ size range relaxes the need to isolate ultralong genomic DNA. Second, increased accuracy allows for more stringent alignment and overlap comparisons, greatly reducing the compute time and cost while improving assembly results by recognizing fine-grained repeat and haplotype phase information. Third, familiar tools like GATK that were developed for accurate short reads are readily applied to CCS reads.

Variant calling and assembly with CCS reads perform well down to 15-fold coverage, which offsets the current reduced throughput per run compared to noisy long reads. Ultimately more unique reads per run (5-10x is expected) and increased read length to allow larger fragment sizes will facilitate rapid, population-scale analysis of full genomes with CCS reads to improve human health. 


\section{Methods}

\section{CCS Library Preparation}

PacBio library preparation and sequencing was performed on the human reference genome sample HG002 obtained from NIST. Genomic DNA was sheared using the Megaruptor $\circledast$ from Diagenode with a long hydropore cartridge and a $20 \mathrm{~kb}$ shearing protocol. Prior to library preparation, the size distribution of the sheared DNA was characterized on the Agilent 2100 BioAnalyzer System using the DNA 12000 kit. A sequencing library was constructed from this sheared genomic DNA using the SMRTbell $^{\text {TM }}$ Template Prep Kit v 1.0 (Pacific Biosciences Ref. No. 100-259-100). In order to tighten the size distribution of the SMRTbell ${ }^{\mathrm{TM}}$ library, the sample was separated into $3 \mathrm{~kb}$ fractions using the SageELF System from Sage Science. Fractions having the desired size distribution ranges were identified on the Agilent 2100 BioAnalyzer using the DNA 12000 kit (Supplementary Figure 1). The fraction centered at $15 \mathrm{~kb}$ was used for sequencing.

\section{Sequencing}

Sequencing reactions were performed on the PacBio Sequel System with the Sequel Sequencing Kit 3.0 chemistry. The samples were pre-extended without exposure to illumination for 12 hours to enable the polymerase enzymes to transition into the highly processive strand-displacing state and sequencing data was collected for 24 hours to ensure suitably long read lengths. Average subread yield was $35.3 \pm 6.2 \mathrm{~Gb}$ over 39 SMRT Cells with average polymerase read length of $100.0 \mathrm{~kb}$.

Consensus reads ("CCS reads") were generated using the ccs software version 3.0.0 (https://github.com/pacificbiosciences/unanimity/) with --minPasses 3 -minPredictedAccuracy 0.99 --maxLength 21000. Average run time is 3,035 CPU core hours per SMRT Cell (118,365 total). The total CCS read yield was $89 \mathrm{~Gb}$ $(2.3 \pm 0.4 \mathrm{~Gb}$ per SMRT Cell), with read length of $13.5 \pm 1.2 \mathrm{~kb}$.

\section{Read Mapping}

Reads were mapped to the GRCh37 human reference genome, specifically the hs37d5 build from the 1000 Genome Project ${ }^{44}$. CCS reads were mapped with pbmm2 version 0.10 .0 (https://github.com/PacificBiosciences/pbmm2) with--preset CCS. NGS reads were mapped with minimap $2^{33}$ version $2.14-r 883$ with $-x$ sr.

\section{Measuring HG002 Concordance}

To measure concordance to HG002, alignments to GRCh37 were evaluated at positions within GIAB v3.3.2 benchmark high-confidence regions that have no high-confidence variant call ${ }^{25}$. Concordance $=M /(M+X+D+I)$ where $M$ is the number of matches, $X$ is the number of mismatches, $D$ is the number of deletion basepairs, and I is the number of insertion basepairs. Phred $=-10 * \log _{10}(1$-Concordance). Reads with perfect concordance are assigned a Phred score of $1+\log _{10}$ (ReadLength) . 
A deleted basepair is considered a homopolymer deletion when it matches the preceding or following basepair in the reference genome. An insertion is considered a homopolymer insertion when the basepairs of the insertion are identical and match either the preceding or following basepair in the reference genome.

\section{Coverage by [GC] content}

To measure coverage by local [GC] content, bedtools ${ }^{45}$ version 2.27 .1 was used to divide the GRCh37 reference genome into 500 bp windows (bedtools makewindows -w 500) and then to calculate the [GC] content (bedtools nuc) and average coverage (bedtools coverage -mean) of each window.

\section{Reference-independent Quality Evaluation}

The Dazzler suite (https://dazzlerblog.wordpress.com/) was used to evaluate the accuracy of the CCS reads without relying on a reference genome. Briefly, daligner ${ }^{26}$ was used to produce all local alignments longer than $1 \mathrm{~kb}$ between pairs of CCS reads. Each CCS subject read was partitioned into 100 bp panels, within which its coverage by and concordance to aligned target reads was calculated. Panels with a concordance in the worst $0.1 \%$ were considered low quality. Abrupt breaks in read-to-read alignments were used to estimate library artifacts like chimeric molecules and missing adapters.

\section{Mappability of CCS and NGS Reads}

To compare with the mappability of $13.5 \mathrm{~kb}$ CCS reads, a coverage-matched (89 $\mathrm{Gb})$ set of $2 \times 250 \mathrm{bp}$ Illumina HiSeq 2500 reads for HG002 were obtained from $\mathrm{GIAB}^{24}$ and mapped to GRCh37 with minimap $2^{33}$ version 2.14-r883 with $-x$ sr.

A genome position is considered mappable if it is covered by alignments for at least ten reads at a specified mapping quality or higher, which was evaluated using bedtools bamtobed and bedtools genomecov -bga. Gaps ("N" basepairs in the reference) were excluded.

Previously-reported NGS problem exons ${ }^{27}$ were considered mappable if every basepair in the exon is covered by a read at mapping quality of 60 .

\section{HLA Typing}

The HLA-A and HLA-DPA1 genes were typed by comparing the sequence of CCS reads that span the genes to entries in the IMGT database ${ }^{46}$ version 3.19.0.

\section{Small Variant Detection and Benchmarking}

To develop a workflow for calling variants in CCS reads with GATK ${ }^{29}$ HaplotypeCaller v4.0.6.0, different values of the HaplotypeCaller parameter--pcr-indel-model and VariantFiltration parameter - -filter-expression were considered to maximize SNV and indel F1 without excessive complication, starting from the GATK best practices for hard filtering. In the end, HaplotypeCaller was run on reads with a 
minimum mapping quality of 60 using allele-specific annotations (--annotationgroup AS_StandardAnnotation) and--pcr_indel_model AGGRESSIVE. Autosomes and the pseudo-autosomal regions (PARs) on chromosome $X$ were called with - - ploidy 2; chromosome $Y$ and the non-PAR regions of chromosome $X$ were called with - -ploidy 1 . Multi-allelic variant sites were split into separate entries for filtration with a custom script (https://gist.github.com/williamrowell/16cd89fcb23ab9f11a7bd387c308d29d). SNVs were filtered using GATK VariantFiltration with - -filter_expression of $A S \_Q D<2.0$ for SNVs and indels longer than $1 \mathrm{bp}$, and $\bar{A} S_{2} \mathrm{QD}<5.0$ for $1 \mathrm{bp}$ indels. A similar pipeline was used to call variants in coverage-matched $2 \times 151$ bp Illumina NovaSeq reads with a few differences: a minimum mapping quality of $20,--p c r-$ indel-model NONE, --standard_min_confidence_threshold_for_calling 2.0 , and no variant filtration.

A Google DeepVariant model for CCS reads was generated as previously reported 30 using DeepVariant version 0.7.1. Briefly, models were trained using CCS reads for chromosomes 1-19 and the HG002 GIAB v3.3.2 benchmark. A single model was selected based on performance in chromosomes 21 and 22 to avoid overfitting. Neither training nor model selection considers chromosome 20 , which is available for accuracy evaluations. To support long reads, local reassembly is disabled for DeepVariant with CCS reads. The wgs_standard model version 0.7 .1 was used to call variants in NovaSeq reads and to apply a model trained on Illumina reads to CCS reads.

To incorporate long-range haplotype information, DeepVariant was modified to produce pileups with reads sorted by the BAM haplotype ("HP") tag. Haplotype information was added to the pbmm2 CCS alignments using WhatsHap v0.17 (what shap haplotag) with the trio-phased variant calls from GIAB (ftp://ftptrace.ncbi.nlm.nih.gov/giab/ftp/data/AshkenazimTrio/analysis/NIST MPI whatshap 082 32018/RTG.hg19.10x.trio-whatshap.vcf.gz or https://bit.ly/2R73grR). A new DeepVariant model then was trained as described above.

Small variant callsets were benchmarked against the GIAB v3.3.2 HG002 set $^{25}$ by vcfeval $^{47}$ (https://github.com/RealTimeGenomics/rtg-tools) with no partial credit run through hap.py version 0.3.10 (https://github.com/lllumina/hap.py). Only PASS calls were considered.

\section{Phasing Small Variants}

Small variant calls were phased using WhatsHap v0.17 (whatshap phase). The number of switch and Hamming errors was computed against trio-phased variant calls from GIAB using whatshap compare.

To model the phase blocks achievable with a given read length, cuts were introduced between heterozygous variants in the GIAB trio-phased variant callset that are 
separated by more than the read length, which effectively assumes that adjacent heterozygous variants separated by less than the read length can be phased.

Structural Variant Detection

pbsv version 2.1.0 (https://github.com/PacificBiosciences/pbsv) was run on pbmm2 CCS read alignments. The pbsv discover stage was run separately per chromosome with tandem repeat annotations (https://github.com/PacificBiosciences/pbsv/tree/master/annotations) passed with -tandem-repeats. The pbsv call stage was run on the full genome.

Sniffles version 1.0.10 was run on pbmm2 CCS reads alignments with $-s \quad 3$-skip_parameter_estimation and with the variant sequence obtained from reads.

Structural variants in the maternal and paternal Canu and FALCON assemblies from CCS reads (see "De novo Assembly") were called using a previously described workflow ${ }^{48}$. Briefly, contigs were mapped to GRCh37 using minimap2 --paf-nohit --cxasm5 --cs -r2k; variants were called with paftools.js calli3; maternal and paternal variants were concatenated; and indel calls at least 30 bp were retained.

An integrated callset was produced from the pbsv, Sniffles, and paftools/Canu callsets using SURVIVOR ${ }^{49}$ and custom scripts. Two calls were considered supporting if the calls had the same structural variation type, a start position with $1 \mathrm{~kb}$, and a difference in length less than $5 \%$. One call from each matching set was retained with precedence given to pbsv, then Sniffles, and then paftools. Because pbsv and Sniffles have poor sensitivity for calls larger than $1 \mathrm{~kb}$, all non-matched calls from paftools that are larger than $1 \mathrm{~kb}$ were retained.

NovoAlign (http://www.novocraft.com) alignments to GRCh37 of 300-fold coverage of HG002 with 2×250bp Illumina HiSeq 2500 reads were obtained from GIAB. Structural variants were called with Manta ${ }^{34}$ version 1.4 .0 with all coverage, and Delly ${ }^{35}$ version 0.7.6 with coverage subsampled to 30 -fold using samtools view -b - 0.1 .

Structural variant callsets on 10X Genomics reads from LongRanger version 2.2 were obtained from GIAB (ftp://ftp-

trace.ncbi.nlm.nih.gov/giab/ftp/data/AshkenazimTrio/analysis/10XGenomics Chromium Genome LongRanger2.2 Supernova2.0.1 04122018/ or https://bit.ly/2Mtj084). Insertion and deletion variants at least $30 \mathrm{bp}$ were combined from the sequenceresolved indels and large deletion calls (NA24385_LongRanger_snpindel.vcf.gz, NA24385_LongRanger_sv_deletions.vcf.gz). Another callset was produced using paftools on the diploid Supernova 2.0.1 assembly as described above.

Structural variant callsets were benchmarked against the GIAB v0.6 HG002 structural variant set (ftp://ftp- 
trace.ncbi.nlm.nih.gov/giab/ftp/data/AshkenazimTrio/analysis/NIST SVs Integration v0. $\underline{6}$ or https://bit.ly/2T7iLBX) using Truvari (https://github.com/spiralgenetics/truvari) commit 600 b4ed7 modified to allow a single variant in the benchmark set to support multiple variants in the callset. Truvari was run with $-r 1000-\mathrm{p} 0.01$-multimatch --includebed HG002_SVs_Tier1_v0.6.bed -c HG002_SVs_Tier1_v0.6.VCf.gz. The $-\bar{p}$ option was used to disable sequence checks for callsets that report symbolic alleles instead of sequence-resolved calls (LongRanger, Delly).

\section{De novo Assembly}

Mixed haplotype assemblies were produced using all CCS reads. Canu ${ }^{38}$ version 1.7.1 was run with $-\mathrm{p}$ asm genomeSize=3.1g correctedErrorRate=0.015 ovlMerThreshold=75 batoptions="-eg 0.01 -eM 0.01 -dg 6 -db 6 -dr 1 -ca 50 -cp 5" -pacbio-corrected. FALCON ${ }^{37}$ kit version 1.2.0 was run with ovlp_HPCdaligner_option $=-\mathrm{v}-\mathrm{B} 128$-M24 -k24 -h1024 -e.97 -12500 -s100, ovlp_DBsplit_option $=-s 400$, and overlap_filtering_setting = --max-diff $90--\max -\operatorname{cov} 120--m i n-c o v 2$. Wtdbg2 (https://github.com/ruanjue/wtdbg2) version 2.2 was run with -k 0 -p 21 -AS 4 -s 0.5 -e 2 -K 0.05 and followed by wtdbg2-cns.

CCS reads from HG002 were "trio binned" as maternal, paternal, or unassigned as previously described ${ }^{39}$. Briefly, 2×250 bp Illumina HiSeq 2500 reads for the father (HG003/NA24149) and mother (HG004/NA24143) of HG002 were obtained from GIAB. Sequence k-mers unique to the mother or father were identified and used to categorize CCS reads (https://github.com/skoren/triobinningScripts), using k-mer size of 21, 51, and 91 and excluding k-mers that occur 25 times or fewer. The maternal and unassigned reads were used for the "maternal" assemblies; paternal and unassigned reads were used for the "paternal" assemblies.

The maternal and paternal assemblies were generated with Canu and wtdbg2 using the same software version and same options as for the mixed haplotype assembly. For the maternal and paternal assemblies, FALCON version 0.7 was run with

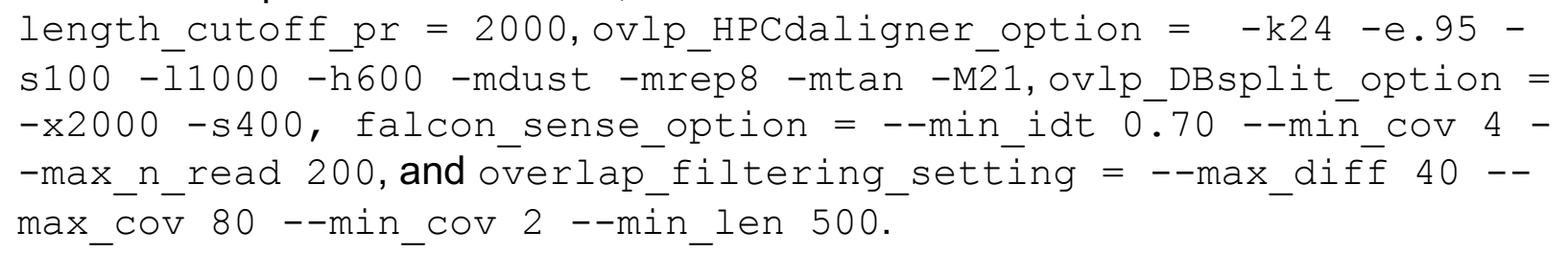

The maternal and paternal Canu assemblies were polished with Arrow version 2.2.2 run through ArrowGrid (https://github.com/skoren/ArrowGrid) using subreads that correspond to the CCS reads used for each assembly. The maternal and paternal FALCON assemblies were polished with Arrow version 2.2.2 using all subreads. 


\section{Assembly Evaluation}

For each assembly, contigs were broken into $100 \mathrm{~kb}$ chunks with remainders shorter than $100 \mathrm{~kb}$ ignored. The chunks were aligned to GRCh37 using minimap2 --eqx $\mathrm{x}$ asm5, and primary alignments that span at least $50 \mathrm{~kb}$ in the reference at higher than $50 \%$ identity were retained. The concordance of each chunk was evaluated just as for CCS reads (see "Measuring HG002 Concordance"). The overall assembly concordance was calculated as the average concordance of the 100 kb chunks.

Gene completeness was measured using $\mathrm{BUSCO}^{50}$ version 3.0.2 using the Mammalia ODB9 gene set. The single plus duplicated gene count in the BUSCO summary is reported. For a human-specific measure of completeness, we calculated the fraction of single-copy human genes that remain single-copy in each assembly. The human transcript sequences from ENSEMBL ${ }^{51}$ build r94 were mapped to each assembly with minimap2 - Cx splice -B 4 -O 4,34 - C9 -uf --cs and evaluated with paftools.js asmgene $-i \quad 0.98$, which retains the longest of overlapping transcripts, and counts a transcript hit if $99 \%$ of the transcript sequence maps at $98 \%$ identity or higher. A single-copy transcript has exactly one hit. Counts are normalized to the number of transcripts that are considered single-copy by these criteria in GRCh38 (GCA_000001405.15).

To measure the number of segmental duplications spanned by each assembly, the assemblies were processed with segDupPlots ${ }^{42}$ (https://github.com/mvollger/segDupPlots), which maps contigs to GRCh38 and considers a segmental duplication to be spanned by the assembly if a contig alignment extends through the segmental duplication with at least $50 \mathrm{~kb}$ on each flank.

\section{Model of Assembly Contiguity}

To predict assembly contiguity at different read lengths and read accuracies, a previously described model ${ }^{21}$ was updated with improvements for high-accuracy reads. Briefly, all repeat annotations for GRCh38 were downloaded from the UCSC Genome Browser. Repeat identity was defined as by each track except for: the nested repeat track where identity was $50+50 *$ score/1000, RepeatMasker where identity was 1((mismatches + deleted + inserted $) / 1000)$, and microsat and windowmasker/sdust which does not define identity and thus was treated as 100\%. Gaps were included as 100\% identity repeats. Additional repeats were added from self-matches using MashMap ${ }^{52}$ (https://github.com/marbl/MashMap).

The assembly contiguity was predicted based on the ability to resolve repeats. At a given percent identity, repeats below that identity were excluded and remaining repeats separate by $15 \mathrm{bp}$ or fewer were merged. Then, cuts were introduced at each at repeats of each given length, and assembly NG50 was calculated assuming that contigs end at each cut. 


\section{Coverage Titration}

To evaluate the performance of variant calling and assembly at different coverage levels, CCS reads were downsampled from the 28-fold dataset and processed. For small variant calling, alignments were subsampled in DeepVariant version 0.7.1 from $4 \%$ to $100 \%$ in steps of $3 \%$. Variants were called on each subsample using the DeepVariant CCS model. Precision and recall for SNVs and indels were evaluated with hap.py as described above (see "Small Variant Detection and Benchmarking"). For phasing, alignments were subsampled (samtools view $-s$ ) at rates from $10 \%$ to $100 \%$ in steps of $10 \%$. The DeepVariant callset from the full 28 -fold coverage data was phased using WhatsHap v0.17 (whatshap phase) with the subsampled alignments. For structural variants, alignments were subsampled (samtools view -s) at rates from $10 \%$ to $100 \%$ in steps of $10 \%$. Variants were called on the subsampled alignments with pbsv version 2.1.0 and benchmarked with Truvari as described above (see "Structural Variant Detection"). For assembly, reads were subsampled at rates from $10 \%$ to $100 \%$ in steps of $10 \%$. Sampling was performed based on read name ( $10 \%$ sample is reads that end in $0,20 \%$ is reads that end in $0-1$, and so on). Assembly of subsamples reads was performed with wtdbg2 version 2.2 and benchmarked as described above (see "De novo Assembly" and "Assembly Evaluation").

\section{Revising and Expanding Genome in a Bottle Benchmarks}

Discrepancies between the GIAB v3.3.2 small variant benchmark and the DeepVariant callset from haplotype-sorted CCS reads were identified with vcfeval and hap.py.

Discrepancies between the GIAB v0.6 structural variant benchmark and the integrated structural variant callset from CCS reads were identified with Truvari. A sample of 60 small variant and 40 structural variant discrepancies were selected for manual curation by random sampling across discrepancy types (false positive, false negative, genotype difference), variant types (SNV, indel, insertion structural variant, and deletion structural variant), both inside and outside homopolymers and tandem repeats. Curators evaluated variants in IGV along with alignments of CCS reads, 10X Genomics reads, Illumina short reads, and Illumina reads from a $6 \mathrm{~kb}$ mate pair library, all obtained from GIAB. The benchmark error rate was estimated by variant type and discrepancy type and used to extrapolate from the sample to the number of errors in the full GIAB benchmark. Confidence intervals were calculated assuming a binomial distribution. 
bioRxiv preprint doi: https://doi.org/10.1101/519025; this version posted January 23, 2019. The copyright holder for this preprint (which was not certified by peer review) is the author/funder, who has granted bioRxiv a license to display the preprint in perpetuity. It is made available under aCC-BY-NC-ND 4.0 International license.

\section{Data Availability}

CCS reads and alignments to GRCh37 are available at ftp://ftp-

trace.ncbi.nlm.nih.gov/giab/ftp/data/AshkenazimTrio/HG002 NA24385 son/PacBio CC $\underline{\mathrm{S} 15 \mathrm{~kb} /}$ or https://bit.ly/2RW1b3l. 


\section{Figures}

Figure 1

a

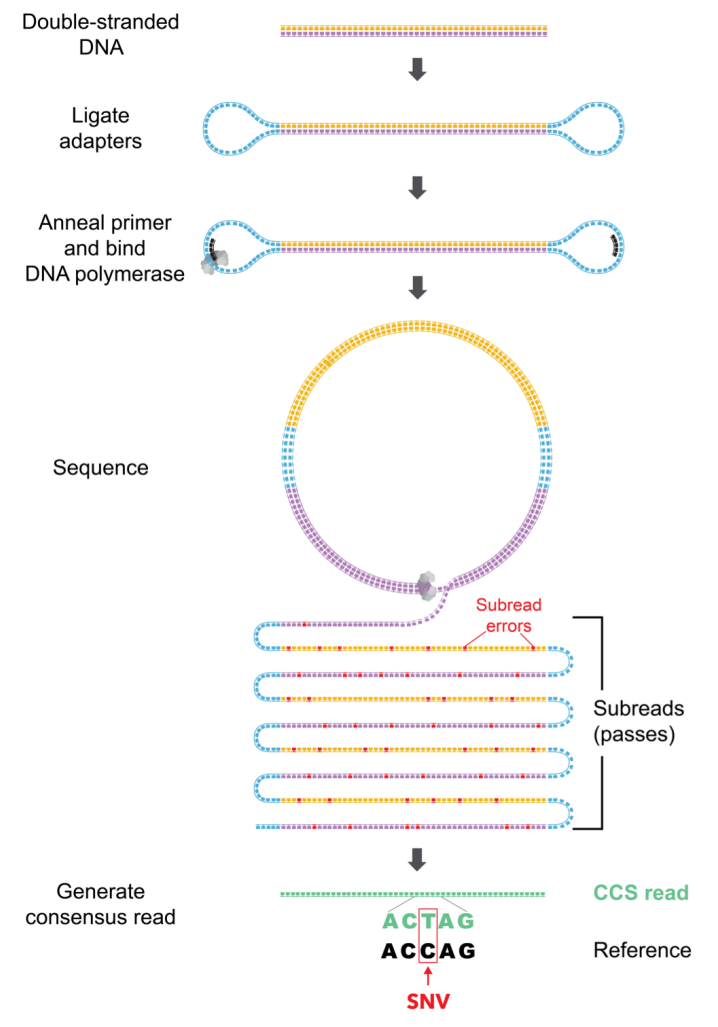

b

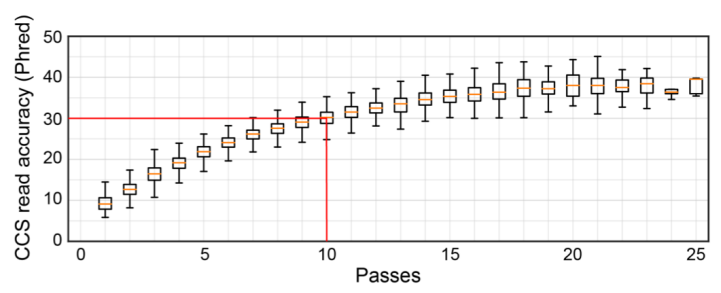

C
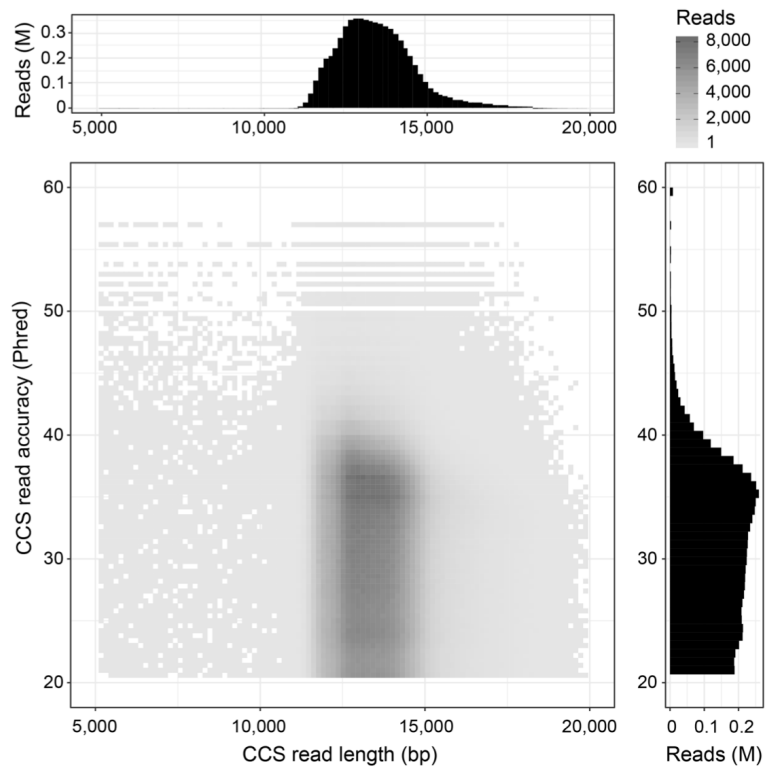

Figure 1. Sequencing HG002 with highly-accurate, long reads. (a) Circular consensus sequencing (CCS) derives a consensus read from multiple passes of a single template molecule, producing accurate reads from noisy individual subreads (passes). (b) Predicted accuracy of CCS reads with different numbers of passes, for sequencing of the human male HG002. At 10 passes, the median read achieves Q30 predicted accuracy. (c) Length and predicted accuracy of CCS reads. 
Figure 2

a

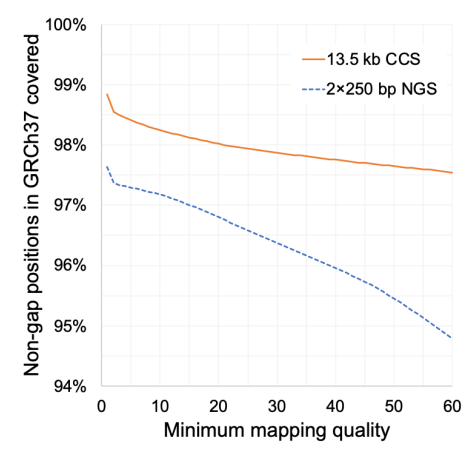

b

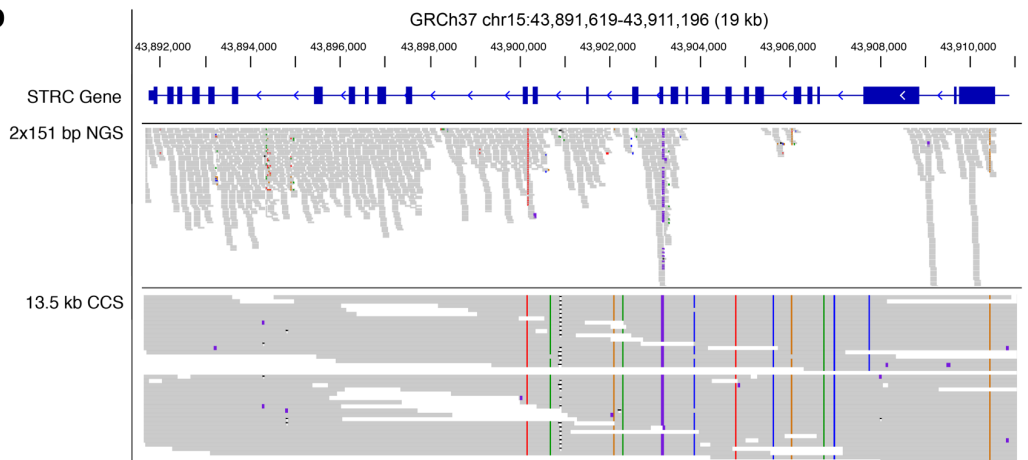

c

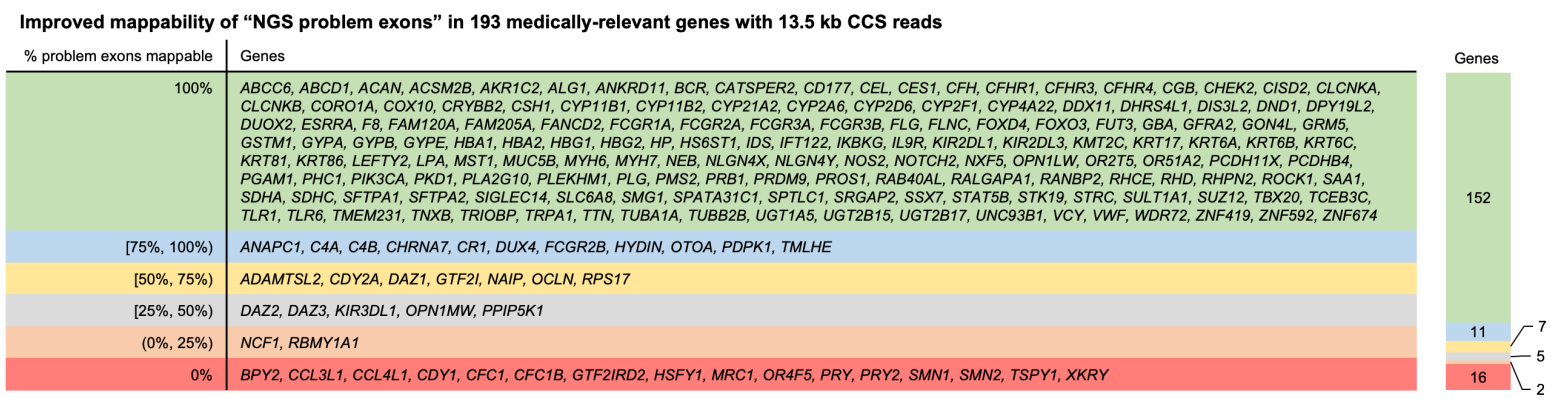

Figure 2. Mappability of the human genome with CCS reads. (a) Percentage of the non-gap GRCh37 human genome covered by at least 10 reads from 28 -fold coverage NGS (2×250 bp) and CCS (13.5 kb) datasets at different mapping quality thresholds. (b) Coverage of the congenital deafness gene STRC in HG002 with $2 \times 151$ bp NGS (NovaSeq) reads and $13.5 \mathrm{~kb}$ CCS reads at a mapping quality threshold of 10 . (c) Improvement in mappability with $13.5 \mathrm{~kb}$ CCS reads for 193 human genes previously reported as medically-relevant and problematic to map with NGS reads ${ }^{27}$. 
Figure 3

a

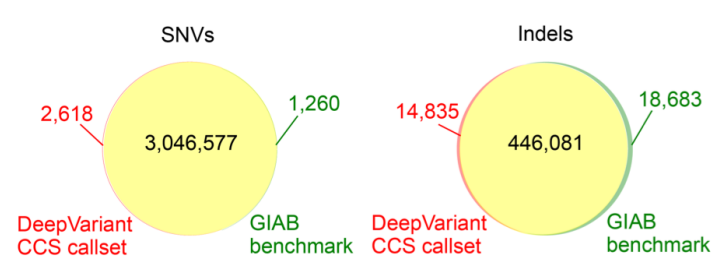

C

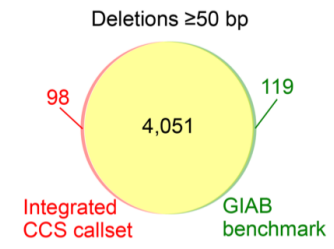

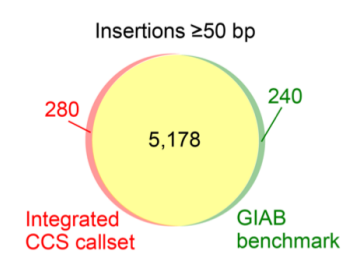

b

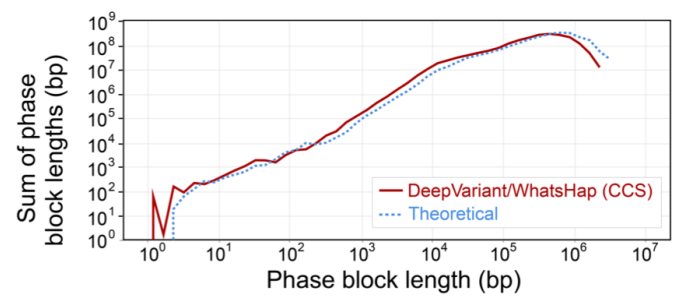

d

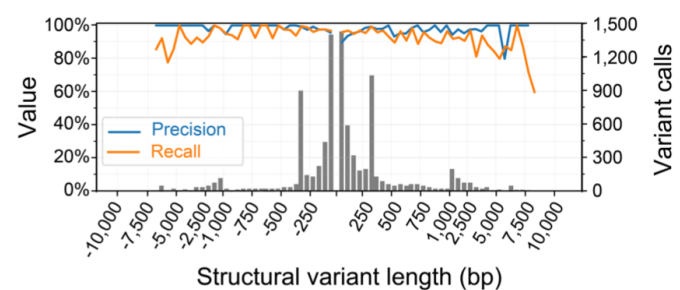

Figure 3. Variant calling and phasing with CCS reads. (a) Agreement of DeepVariant SNV and indel calls with Genome in a Bottle v3.3.2 benchmark measured with hap.py. (b) Phasing of heterozygous DeepVariant variant calls with WhatsHap, compared to theoretical phasing of HG002 with $13.5 \mathrm{~kb}$ reads. (c) Agreement of integrated CCS structural variant calls with the Genome in a Bottle v0.6 structural variant benchmark measured with Truvari, (d) by variant length. Negative length indicates a deletion; positive length indicates an insertion. The histogram bin size is 50 bp for variants shorter than $1 \mathrm{~kb}$, and $500 \mathrm{bp}$ for variants $>1 \mathrm{~kb}$. 
Figure 4

a

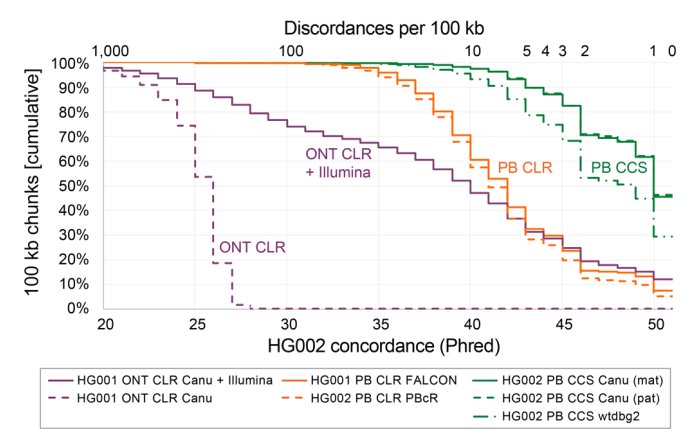

b

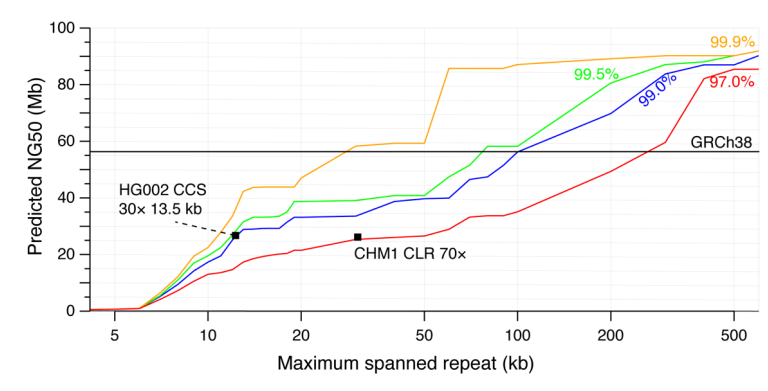

Figure 4. Impact of read accuracy on de novo assembly. (a) The concordance of seven assemblies to the Genome in a Bottle HG002 benchmark (Supplementary Table 7). Contigs longer than $100 \mathrm{~kb}$ were segmented into $100 \mathrm{~kb}$ chunks and aligned to GRCh37. Concordance was measured per chunk, and chunks with no discordances were assigned concordance of Q51. PB=PacBio, ONT=Oxford Nanopore, $C L R=$ continuous ("noisy") long reads. (b) Predicted contiguity of a human assembly based on ability to resolve repeats of different lengths (x-axis) and percent identities (colored lines) ${ }^{21}$. The solid line indicates the contiguity of GRCh38. The $97.0 \%$ identity line is representative of CLR assemblies using standard read-to-read error correction. The points show example CCS and CLR ${ }^{43}$ assemblies using Canu. Repeat identity and length are proxies for read accuracy and length. 


\section{Tables}

\section{Table 1}

\begin{tabular}{|c|c|c|c|c|c|c|c|}
\hline \multirow[b]{2}{*}{ Platform } & \multirow[b]{2}{*}{ Variant caller (training model) } & \multicolumn{3}{|c|}{ SNVs } & \multicolumn{3}{|c|}{ Indels } \\
\hline & & Precision & Recall & $\mathrm{F} \mathbf{1}^{\wedge}$ & Precision & Recall & F1 \\
\hline Illumina (NovaSeq) & DeepVariant (Illumina model) & $\underline{99.960 \%}$ & $\underline{99.940 \%}$ & $\underline{99.950 \%}$ & $99.633 \%$ & $99.413 \%$ & $99.523 \%$ \\
\hline PacBio (CCS) & DeepVariant (CCS model) & $\underline{99.914 \%}$ & $\underline{99.959 \%}$ & $\underline{99.936 \%}$ & $96.901 \%$ & $95.980 \%$ & $96.438 \%$ \\
\hline PacBio (CCS) & DeepVariant (haplotype-sorted CCS model) & $\underline{99.904 \%}$ & $\underline{99.963 \%}$ & $\underline{99.934 \%}$ & $97.835 \%$ & $97.141 \%$ & $97.486 \%$ \\
\hline Illumina (NovaSeq) & GATK HaplotypeCaller (no filter) & $99.852 \%$ & $99.910 \%$ & $99.881 \%$ & $99.371 \%$ & $99.156 \%$ & $99.264 \%$ \\
\hline PacBio (CCS) & GATK HaplotypeCaller (hard filter) & $99.468 \%$ & $99.559 \%$ & $99.513 \%$ & $78.977 \%$ & $81.248 \%$ & $80.097 \%$ \\
\hline
\end{tabular}

Table 1. Performance of small variant calling with CCS reads. Precision, recall, and F1 of small variant calling measured against the Genome in a Bottle v3.3.2 benchmark using hap.py. Bold indicates the highest value in each column. Underline indicates a value higher than the GATK HaplotypeCaller run on 30 -fold Illumina NovaSeq reads. Coverage is 28 -fold for PacBio CCS and 30 -fold for Illumina NovaSeq. Rows are sorted ("A") based on F1 for SNVs. 


\section{Table 2}

\begin{tabular}{|c|c|c|c|c|c|c|c|c|c|c|}
\hline Haplotype & Assembler & $\begin{array}{r}\text { Total } \\
\text { size } \\
\text { (Gb) }\end{array}$ & Contigs & $\begin{array}{l}\text { N50 } \\
\text { (Mb) }\end{array}$ & $\begin{array}{r}\text { NG50 } \\
\text { (Mb) }\end{array}$ & $\begin{array}{l}\text { Max } \\
\text { (Mb) }\end{array}$ & $\begin{array}{r}\text { E-size }{ }^{53} \\
(\mathbf{M b})\end{array}$ & $\begin{array}{r}\text { HG002 } \\
\text { concordance } \\
\text { (Phred) }\end{array}$ & $\begin{array}{r}\text { BUSCO } \\
\text { genes }\end{array}$ & $\begin{array}{r}\text { RefSeq } \\
\text { genes }\end{array}$ \\
\hline Mixed & Canu & 3.42 & 18,006 & 22.78 & 25.02 & 108.46 & 30.16 & 31.1 & $92.3 \%$ & $93.2 \%$ \\
\hline Mixed & FALCON & 2.91 & 2,541 & 28.95 & 24.51 & 110.21 & 38.04 & 25.8 & $87.6 \%$ & $97.6 \%$ \\
\hline Mixed & wtdbg2 & 2.79 & 1,554 & 15.43 & 12.62 & 84.67 & 22.61 & 44.6 & $94.2 \%$ & $96.1 \%$ \\
\hline Maternal & Canu* & 3.04 & 5,854 & 18.02 & 17.04 & 48.81 & 19.78 & 47.2 & $94.1 \%$ & $98.1 \%$ \\
\hline Maternal & FALCON $^{*}$ & 2.80 & 924 & 19.99 & 15.54 & 74.33 & 24.07 & 43.5 & $95.1 \%$ & $97.8 \%$ \\
\hline Maternal & wtdbg2 & 2.75 & 2,637 & 12.10 & 9.29 & 66.34 & 16.55 & 43.5 & $93.8 \%$ & $95.6 \%$ \\
\hline Paternal & Canu* & 2.96 & 6,868 & 16.14 & 14.90 & 64.83 & 20.19 & 47.7 & $93.4 \%$ & $98.2 \%$ \\
\hline Paternal & FALCON* & 2.70 & 1,489 & 16.40 & 14.06 & 95.34 & 25.61 & 43.5 & $93.6 \%$ & $97.7 \%$ \\
\hline Paternal & wtdbg2 & 2.67 & 1,444 & 13.96 & 10.86 & 50.51 & 15.36 & 42.1 & $92.6 \%$ & $95.3 \%$ \\
\hline
\end{tabular}

Table 2. Statistics for de novo assembly of CCS reads. The "mixed" haplotype assemblies use all reads. The "maternal" and "paternal" assemblies use parent-specific reads from trio binning plus unassigned reads. HG002 concordance is measured against the Genome in a Bottle benchmark. BUSCO gene completeness uses the Mammalia ODB9 gene set. RefSeq genes is the percentage of genes from Ensembl R94 that are full-length, single-copy in the assembly relative to the full-length, singlecopy count for GRCh38. Contigs shorter than $13 \mathrm{~kb}$ were excluded from genome size and contiguity measurements; contigs shorter than $100 \mathrm{~kb}$ were excluded from the concordance measurement. "*" indicates polishing with Arrow. 


\section{Author Contributions}

AMW, DRR, MWH, and PP designed the study. DRR and PP developed the sample preparation protocol and performed sample preparation. DRR, $P P$, and $Y Q$ performed sequencing. AC, AK, CSC, MAD, and $P C$ adapted the algorithms and implementation of DeepVariant. AC, AF, AK, AMP, AMW, AT, CSC, DRR, FJS, GM, GTC, HL, JE, JMZ, JR, MA, MAD, MCS, MM, NDO, PC, PP, RJH, SK, TM, and WJR performed analysis. AC, AMP, CSC, DRR, FJS, JMZ, MAD, MCS, and MWH supervised analysis. AC, AMW, DRR, GM, JMZ, PP, RH, SK, and WJR wrote the manuscript. All authors reviewed and approved the final manuscript.

\section{Acknowledgements}

We would like to thank John R. Harting for assistance with HLA typing and Kristin A. Robertshaw for figure generation.

SK and AMP were supported by the Intramural Research Program of the National Human Genome Research Institute, National Institutes of Health. This work utilized the computational resources of the NIH HPC Biowulf cluster (https://hpc.nih.gov).

This work was supported by NIH grant 1R01HG010040 to HL and NSFC grants 31571353 and 31822029 to JR. MCS is funded by the National Science Foundation (DBI-1350041) and National Institutes of Health (R01-HG006677). FJS and MM are funded by NIH grant UM1 HG008898.

This work utilized computational resources of DNAnexus and Google to apply DeepVariant to CCS reads.

Certain commercial equipment, instruments, or materials are identified to specify adequate experimental conditions or reported results. Such identification does not imply recommendation or endorsement by the National Institute of Standards, nor does it imply that the equipment, instruments, or materials identified are necessarily the best available for the purpose.

\section{Competing Financial Interests}

AMW, AT, DRR, GTC, MWH, PP, RJH, WJR, and YQ are employees and shareholders of Pacific Biosciences. AC, AK, MAD, and PC are employees and shareholders of Google. AF and CSC are employees and shareholders of DNAnexus. AC is a shareholder and was an employee of DNAnexus for a portion of this work. 


\section{References}

1. DNA Sequencing Costs: Data. National Human Genome Research Institute (NHGRI) Available at: https://www.genome.gov/27541954/dna-sequencing-costsdata/. (Accessed: 7th December 2018)

2. Sanger, F., Nicklen, S. \& Coulson, A. R. DNA sequencing with chain-terminating inhibitors. Proc. Natl. Acad. Sci. U. S. A. 74, 5463-5467 (1977).

3. Smith, L. M. et al. Fluorescence detection in automated DNA sequence analysis. Nature 321, 674-679 (1986).

4. Lander, E. S. et al. Initial sequencing and analysis of the human genome. Nature 409, 860-921 (2001).

5. Venter, J. C. et al. The sequence of the human genome. Science 291, 1304-1351 (2001).

6. Mouse Genome Sequencing Consortium et al. Initial sequencing and comparative analysis of the mouse genome. Nature 420, 520-562 (2002).

7. Arabidopsis Genome Initiative. Analysis of the genome sequence of the flowering plant Arabidopsis thaliana. Nature 408, 796-815 (2000).

8. Ronaghi, M., Karamohamed, S., Pettersson, B., Uhlén, M. \& Nyrén, P. Real-time DNA sequencing using detection of pyrophosphate release. Anal. Biochem. 242, 84-89 (1996).

9. Bentley, D. R. et al. Accurate whole human genome sequencing using reversible terminator chemistry. Nature 456, 53-59 (2008).

10. Shendure, J. et al. Accurate multiplex polony sequencing of an evolved bacterial genome. Science 309, 1728-1732 (2005).

11. McKernan, K. J. et al. Sequence and structural variation in a human genome uncovered by short-read, massively parallel ligation sequencing using two-base encoding. Genome Res. 19, 1527-1541 (2009).

12. Drmanac, R. et al. Human genome sequencing using unchained base reads on self-assembling DNA nanoarrays. Science 327, 78-81 (2010).

13. Rothberg, J. M. et al. An integrated semiconductor device enabling non-optical genome sequencing. Nature 475, 348-352 (2011). 
14. Sedlazeck, F. J., Lee, H., Darby, C. A. \& Schatz, M. C. Piercing the dark matter: bioinformatics of long-range sequencing and mapping. Nat. Rev. Genet. 19, 329346 (2018).

15. Chaisson, M. J. P. et al. Resolving the complexity of the human genome using single-molecule sequencing. Nature 517, 608-611 (2015).

16. Seo, J.-S. et al. De novo assembly and phasing of a Korean human genome. Nature 538, 243-247 (2016).

17. Cretu Stancu, M. et al. Mapping and phasing of structural variation in patient genomes using nanopore sequencing. Nat. Commun. 8, 1326 (2017).

18. Chaisson, M. J. P. et al. Multi-platform discovery of haplotype-resolved structural variation in human genomes. bioRxiv 193144 (2018). doi:10.1101/193144

19. Eid, J. et al. Real-time DNA sequencing from single polymerase molecules. Science 323, 133-138 (2009).

20. Mikheyev, A. S. \& Tin, M. M. Y. A first look at the Oxford Nanopore MinION sequencer. Mol. Ecol. Resour. 14, 1097-1102 (2014).

21. Jain, M. et al. Nanopore sequencing and assembly of a human genome with ultralong reads. Nat. Biotechnol. 36, 338-345 (2018).

22. Travers, K. J., Chin, C.-S., Rank, D. R., Eid, J. S. \& Turner, S. W. A flexible and efficient template format for circular consensus sequencing and SNP detection. Nucleic Acids Res. 38, e159 (2010).

23. Loomis, E. W. et al. Sequencing the unsequenceable: expanded CGG-repeat alleles of the fragile X gene. Genome Res. 23, 121-128 (2013).

24. Zook, J. M. et al. Extensive sequencing of seven human genomes to characterize benchmark reference materials. Sci. Data 3, 160025 (2016).

25. Zook, J. et al. Reproducible integration of multiple sequencing datasets to form high-confidence SNP, indel, and reference calls for five human genome reference materials. bioRxiv 281006 (2018). doi:10.1101/281006

26. Myers, G. Efficient Local Alignment Discovery amongst Noisy Long Reads. in Algorithms in Bioinformatics (eds. Brown, D. \& Morgenstern, B.) 52-67 (Springer Berlin Heidelberg, 2014).

27. Mandelker, D. et al. Navigating highly homologous genes in a molecular diagnostic setting: a resource for clinical next-generation sequencing. Genet. Med. Off. J. Am. Coll. Med. Genet. 18, 1282-1289 (2016). 
28. Ambardar, S. \& Gowda, M. High-Resolution Full-Length HLA Typing Method Using Third Generation (Pac-Bio SMRT) Sequencing Technology. Methods Mol. Biol. Clifton NJ 1802, 135-153 (2018).

29. DePristo, M. A. et al. A framework for variation discovery and genotyping using next-generation DNA sequencing data. Nat. Genet. 43, 491-498 (2011).

30. Poplin, R. et al. A universal SNP and small-indel variant caller using deep neural networks. Nat. Biotechnol. 36, 983-987 (2018).

31. Patterson, M. et al. WhatsHap: Weighted Haplotype Assembly for FutureGeneration Sequencing Reads. J. Comput. Biol. J. Comput. Mol. Cell Biol. 22, 498-509 (2015).

32. Sedlazeck, F. J. et al. Accurate detection of complex structural variations using single-molecule sequencing. Nat. Methods 15, 461-468 (2018).

33. Li, H. Minimap2: pairwise alignment for nucleotide sequences. Bioinformatics $\mathbf{3 4}$, 3094-3100 (2018).

34. Chen, X. et al. Manta: rapid detection of structural variants and indels for germline and cancer sequencing applications. Bioinformatics 32, 1220-1222 (2016).

35. Rausch, T. et al. DELLY: structural variant discovery by integrated paired-end and split-read analysis. Bioinforma. Oxf. Engl. 28, i333-i339 (2012).

36. Garcia, S. et al. Linked-Read sequencing resolves complex structural variants. bioRxiv 231662 (2017). doi:10.1101/231662

37. Chin, C.-S. et al. Phased diploid genome assembly with single-molecule real-time sequencing. Nat. Methods 13, 1050-1054 (2016).

38. Koren, S. et al. Canu: scalable and accurate long-read assembly via adaptive kmer weighting and repeat separation. Genome Res. 27, 722-736 (2017).

39. Koren, S. et al. De novo assembly of haplotype-resolved genomes with trio binning. Nat. Biotechnol. (2018). doi:10.1038/nbt.4277

40. Fungtammasan, A. \& Hannigan, B. How well can we create phased, diploid, human genomes?: An assessment of FALCON-Unzip phasing using a human trio. bioRxiv 262196 (2018). doi:10.1101/262196

41. Chin, C.-S. et al. Nonhybrid, finished microbial genome assemblies from long-read SMRT sequencing data. Nat. Methods 10, 563-569 (2013). 
42. Vollger, M. R. et al. Long-read sequence and assembly of segmental duplications. Nat. Methods (2018). doi:10.1038/s41592-018-0236-3

43. Schneider, V. A. et al. Evaluation of GRCh38 and de novo haploid genome assemblies demonstrates the enduring quality of the reference assembly. Genome Res. 27, 849-864 (2017).

44. 1000 Genomes Project Consortium et al. A global reference for human genetic variation. Nature 526, 68-74 (2015).

45. Quinlan, A. R. BEDTools: The Swiss-Army Tool for Genome Feature Analysis. Curr. Protoc. Bioinforma. 47, 11.12.1-34 (2014).

46. Robinson, J., Soormally, A. R., Hayhurst, J. D. \& Marsh, S. G. E. The IPDIMGT/HLA Database - New developments in reporting HLA variation. Hum. Immunol. 77, 233-237 (2016).

47. Cleary, J. G. et al. Comparing Variant Call Files for Performance Benchmarking of Next-Generation Sequencing Variant Calling Pipelines. bioRxiv 023754 (2015). doi:10.1101/023754

48. Li, H. et al. A synthetic-diploid benchmark for accurate variant-calling evaluation. Nat. Methods 15, 595-597 (2018).

49. Jeffares, D. C. et al. Transient structural variations have strong effects on quantitative traits and reproductive isolation in fission yeast. Nat. Commun. 8, 14061 (2017).

50. Simão, F. A., Waterhouse, R. M., loannidis, P., Kriventseva, E. V. \& Zdobnov, E. M. BUSCO: assessing genome assembly and annotation completeness with single-copy orthologs. Bioinforma. Oxf. Engl. 31, 3210-3212 (2015).

51. Zerbino, D. R. et al. Ensembl 2018. Nucleic Acids Res. 46, D754-D761 (2018).

52. Jain, C., Koren, S., Dilthey, A., Phillippy, A. M. \& Aluru, S. A fast adaptive algorithm for computing whole-genome homology maps. Bioinforma. Oxf. Engl. 34, i748i756 (2018).

53. Salzberg, S. L. et al. GAGE: A critical evaluation of genome assemblies and assembly algorithms. Genome Res. 22, 557-567 (2012). 